\title{
Thin Cloud-fog Cover Removed from Remote Sensing Imagery Based on Stationary Wavelet Transformation
}

\author{
HE Hui, CHEN Hai \\ School of Information Technology \\ Beijing Normal University ZHUHAI \\ Zhuhai, China \\ hhdpczh@aliyun.com, isabelic@163.com
}

\begin{abstract}
A new method based on stationary wavelet transformation and nonlinear gray-scale transformation was proposed to weaken the thin cloud-fog cover of remote sensing imagery in this paper, which can not only to weak the cover of thin cloud-fog effectively with enhanced image resolution but also to preserve the true spectral characteristics of the original imagery and get better classification result. The experimental data was a window of SPOT5 with heavy thin cloud-fog cover acquired from Zhuhai, China. There were comparative experiments with other two existing methods: homomorphism filter and a approach based on
\end{abstract}

\section{INTRODUCTION}

With the advantages of having abundant spectrum information, high spatial resolution and stable performance on geometric positioning, the remote sensing data such as SPOT and Landsat hold important positions in Geo-Science Analyses of Remote Sensing. However, the visible and nearinfrared ones are vulnerable to the effects of weather, for example, the cloud-fog is one of the common noise source) of these remote sensing images. Therefore, It is essential to seek an effective Preprocessing approach to reduce or eliminate the effects deriving from cloud-log, which is not only the foundation of accurate interpretation toward remote sensing imageries, but a significant approach to enhance the effectiveness and usefulness of remote sensing data.

Since the 1970s, to gain more distinct and effective remote sensing imageries, various methods have been tried to eliminate the cloud-fog interference. Up to nowadays, the main approaches to restore the missing information in the cloud-fog-covered areas, can be summarized as follows:

1) Applying the absorption waveband of water vapor to inverse the radiation value, and then eliminating the radiation made by cloud- fog from the original visible waveband [7$10]$.

2) Method based on histogram matching. This method has the shortcomings that sometimes the assumed conditions may not be met, and it is difficult to precisely determine the range of cloud-fog-covered areas.

3) Method based on homomorphic-filtering(HF) or the improved one. This method neglects the fact that Low frequency components not only contain the information of
Laplacian enhancement and histogram shifts. PSNR, average absolute deviation and spectral correlation coefficient were used to evaluate the effect. Furthermore, supervised classification is applied to all the processed images to demonstrate the effectiveness of our integrated method. The results show that the proposed method in this paper performs better. Besides, the spatial details are persisted as more as possible.

Keywords- stationary wavelet transform; thin cloud-fog cover; nonlinear gray-scale transformation; remote sensing imagery

thin cloud-fog, but also a wealth of other background information, with the result that it will weaken other background information of imageries and change the region characteristics.

4) Method using spatial filtering and gray-scale transformation, such as the thin cloud-fog removal and information-enhancing method based on Laplace and histogram transformation.

5) Applying the cloud-fog removal method using multiscale wavelet transformation. Though this method can remove the cloud noise and do a good job in retaining the image details, it still involves in spatial and frequency domain, which may gain inferior processing effects at the case of small cloud-covered area.

6) Applying the cloud-fog removal method based on the mixed pixel interpretation or based on data fusion technique.

Above all, this paper proposes the method based on stationary wavelet transformation and nonlinear gray-scale transformation, fully considering the characteristics of water environment, this method regarding the water body pixel spectrum as the combination of spectra such as water, pollutants, thin cloud-fog, etc. gaining good visual effects and statistical evaluation results, and improving the classification effects. The results of comparison experiments demonstrate that this method is effective to remove the thin cloud-fog in remote sensing imageries. 


\section{THE THIN CLOUD-FOG REMOVAL ALGORITHMS BASED ON THE ENHANCEMENT OF STATIONARY WAVELET TRANSFORMATION}

\section{A. Algorithm description}

The basic ideal of this paper to remove the thin cloud-fog is to lower the mean value of brightness, enhance the contrast in this area and improve the space resolution. The key of the algorithm is the combination of the stationary wavelet transformation and discrete wavelet transformation, the ideal is illustrated in Fig (1)

To make further effects to induce the cloud and enhance the details and contrast of the imageries, this paper introduces nonlinear gray-scale transformation to improve the gray-scale dynamic range of images: enlarging the dynamic range of the buildings on the ground and compacting the one where the cloud information are highly centralized so as to distinguish the features of objects and restore the image details. The imageries among different gray-scale should adopt different functions of gray-scale transformation to enhance the image contract.

\section{B. Algorithm}

Firstly, to retain the high-frequency components of imageries, DWT algorithm is utilized in those imageries that Severe interference from cloud-fog is taken place in corresponding wave-brand: it divides a low resolution imagery into different sub-brand ones, three high-frequency sub-brands(LH,HL and $\mathrm{HH})$ contain the high-frequency components of the imageries as input. To make the information loss occur in the corresponding sub-bands of discrete wavelet transform (DWT), down-sampling is employed in each sub-band. Not needing to take a downsampling, SWT will not cause an information loss. By this point, the method of this paper gets the frequency sub- brand of input imageries through stationary wavelet transform (SWT)[18-19], and adds it and interpolation high-frequency sub-band processed by the SWT to get a correct highfrequency estimation coefficient. Meanwhile, interpolation operation is also done in respectively original input imageries Finally, high resolution output imageries can be acquired through the combination of high-frequency coefficients and the input image using inverse discrete wavelet transformation (IDWT), the details are given in Fig 1. Only simple enhancement process, retaining the objects' information to the maximum, is applied in those wave brand imageries on which the cloud-fog has little effects.

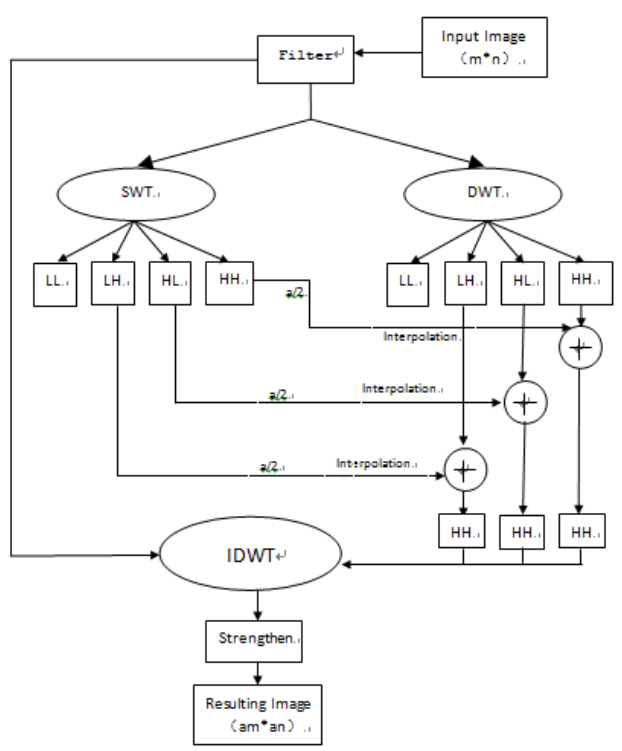

Fig. 1 Technology framework

Because of the important role of nonlinear gray-scale transformation in the process of removing the thin cloud-fog and enhancing the image information, the design of nonlinear function is significant important. To lower the mean value of brightness in cloud-fog-covered areas and enhance the contract in them, combining the features of contract imagery $\mathrm{FH}$, this paper designs the nonlinear function $\mathrm{K}(\mathrm{FL})$, as Equation(1) shows. Besides, nonlinear transformation is also performed on the local mean gray-scale value figure, as is shown in Equation(1).

$$
\begin{aligned}
K\left(F_{L}\right) & =\left\{\begin{array}{lc}
(3 / 160) * F_{L}+2 & F_{L}<=150 \\
(1 / 10) * F_{L}-10 & 150<F_{L}<=170 \\
(3 / 80) * F_{L}+5 / 8 & 170<F_{L}<=210 \\
8.5 & \text { else }
\end{array}\right. \\
F_{L}^{\prime} & = \begin{cases}(2 / 3)^{*} F_{L}+60 & F_{L}<=90 \\
(4 / 15)^{*} F_{L}+96 & 90<F_{L}<=255\end{cases}
\end{aligned}
$$

\section{THE EXPERIMENTS AND ANALYSIS OF THE RESULTS}

This paper selected a $256 \times 256$ pixels area which is largely effected by the cloud-fog from the SPOT5 multispectral remote sensing imagery in Zhuhai City,and carried out experiments in it. Furthermore, sets of comparison research experiments to compare the proposed method in this paper with homomorphic filtering method and the method told by in[3] were conducted. Due to the limit of space, only one set of results is given, as is shown in Fig. 3.

The chosen evaluation indexes are Peak Signal to Noise Ratio(PSNR), which reflects the fidelity of the color remote sensing imagery after a process of removing the cloud-fog noise, and the average deviation, reflecting the definition of imageries. The two are defined as follows: 


$$
P S N R=10 \lg \sum \frac{3 \times 255^{2} \times S U M}{\left((R(x)-r(x))^{2}+(G(x)-g(x))^{2}+(B(x)-b(x))^{2}\right)}
$$

$$
\begin{aligned}
M A E=\frac{1}{255 \times S U M} \sum(|\mathrm{R}(\mathrm{x})-\mathrm{r}(\mathrm{x})|+|\mathrm{G}(\mathrm{x})-\mathrm{g}(\mathrm{x})| \\
+|\mathrm{B}(\mathrm{x})-\mathrm{b}(\mathrm{x})|)
\end{aligned}
$$

Where

$$
f(x)=(R(x), G(x), B(x))
$$

$f^{\prime}(x)=(r(x), g(x), b(x))$ respectively indicate the in imageries and imageries without assessment, SUM stands for the number of pixels. The ideal results may have the following characteristics: The MAE is small and the PSNR is large. The comparison results of comprehensive assessment toward the different methods tested in different data sources can be seen in Table 1.

Besides, to assess the difference of spectral characteristics of the original imageries and the enhanced ones, the relative spectral coefficient is introduced, its definition as shown in Equation(5).

$$
C(f, g)=\frac{\sum\left[\left(f_{i, j}-e_{f}\right) \times\left(g_{i, j}-e_{g}\right)\right]}{\sqrt{\left.\sum_{i, j}\left[\left(f_{i, j}-e_{f}\right)^{2}\right] \times\left(g_{i, j}-e_{g}\right)^{2}\right]}}
$$

$f_{i, j}$ and $g_{i, j}$ respectively indicate the gray-scale value of the two imageries at the point of $(i, j)$. The changing degree can be seen when comparing the relative spectral coefficients of spectral information of multispectral imageries before and after the enhancement, the comparison results as shown in TABLE 2.

TABLE 1. STATISTICS OF COMPREHENSIVE EVALUATION OF THE PROCESSED IMAGERY WITH DIFFERENT METHODS

\begin{tabular}{ccc}
\hline method & $\begin{array}{c}\text { mean atsolute } \\
\text { deviation }\end{array}$ & $\begin{array}{c}\text { peak signal to noise } \\
\text { ratio }\end{array}$ \\
\hline the homomorphic filtering method & 0.4153 & 38.4817 \\
the method told by in [3] & 0.2826 & 41.8651 \\
the proposed method in this paper & 0.2435 & 49.4503 \\
\hline
\end{tabular}

In Fig.2, we can directly see that three methods have their own level of weakening the cloud-fog noise, with the proposed method in this paper being the foremost, especially in those areas which are richest in ground details, such as urban areas and the road network areas.

TABLE 2. COMPARISON OF THE SPECTRAL CORRELATION COEFFICIENT OF DIFFERENT METHODS FOR REMOVING THE THIN CLOUD-FOG COVER

\begin{tabular}{ccccc}
\hline method & B4 & B3 & B2 & Mean value \\
\hline HF & 1 & 1 & 1 & 1 \\
the method[3] & 0.977 & 0.998 & 0.997 & 0.99123 \\
the proposed method & 8 & 4 & 5 & \\
& 0.995 & 0.994 & 0.786 & 0.92553 \\
\hline
\end{tabular}

As is shown in TABLE 1, the values of this table are same with those of visual interpretation, and compared with the other two methods, the MAE values gained in the method of this paper are smaller and the PSNR values are larger, demonstrating that, from a statistical point of view, the processing effects of this paper method are more ideal. Besides, we can also find that this method also does well in retaining the Real spectral characteristics of original imageries, as the lowest average value of spectral correlation coefficient of imageries and original imageries after a thin cloud-fog removal process is 0.9255 , and the imageries before and after processing)/ (the after-processed imageries and original imageries )which were seriously floud-fogeffected in Band 2, got lower relative spectral coefficients. As for those in the wave-band 3 and wave-band 4, less effected by the cloud-fog, retained a good spectral correlation after an enhancement. While those imageries processed with the homomorphic filtering method or the method told by in [3], got higher relative spectral coefficients in each wave-band or in original one. Especially, the coefficients obtained by homomorphic filtering method were up to 1 , which were obviously opposed to facts. Therefore, either from the perspective of the visual effects or statistical evaluation results, the proposed method in this paper had a most ideal performance on removing the cloud-fog.

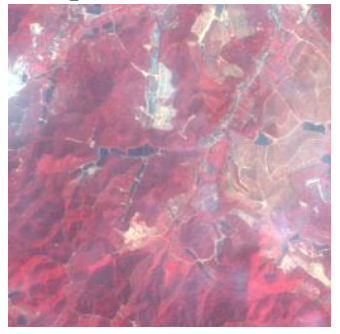

(a)

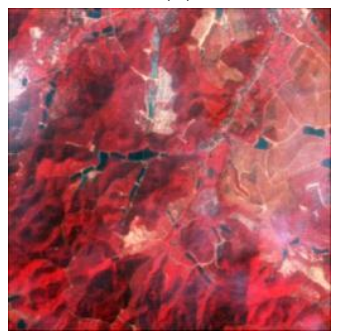

(c)

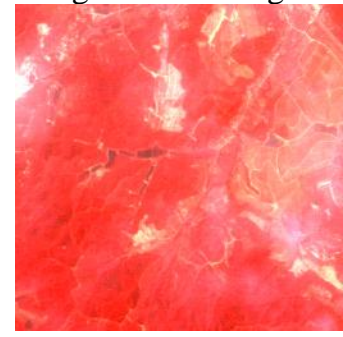

(b)

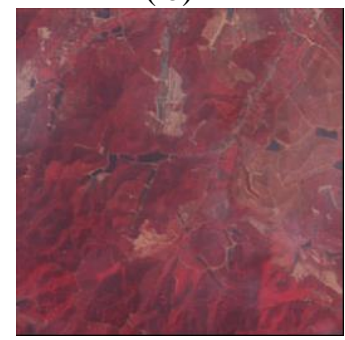

(d)
Fig. 2.Comparison of the resulting images of different methods: (a)original imageries (b)result from the homomorphic filtering method(c)result from the method of in[3] (d)result from the proposed method in paper

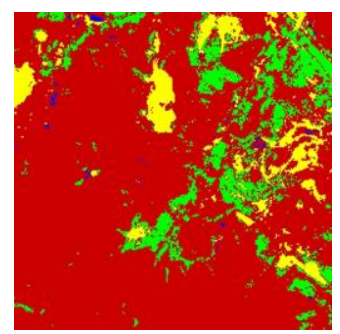

(a)

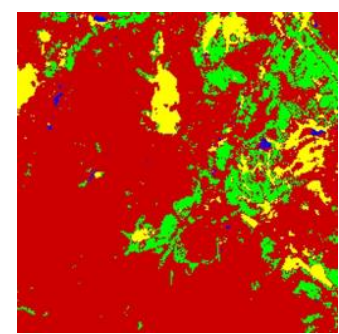

(b) 


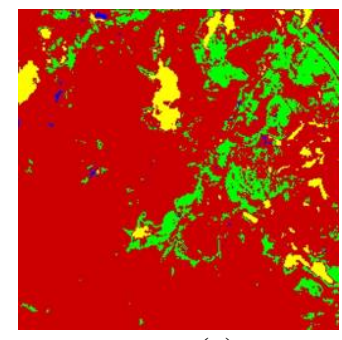

(c)

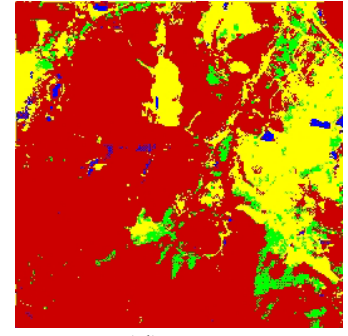

(d) water woods grass buildings

Fig.3.Comparison of the classification images for SPOT imagery: (a)Classification result of original SPOT image;(b)Classification result from the homomorphic filtering method;(c)Classification result from the method told by in[3] ; (d)Classification result from the proposed method in paper

To make further effects to assess the reliability of the results, same training samples were designed to each set of thin cloud-fog removal imageries, which is classified with maximum likelihood estimation (MLE), the results as shown in the Fig.3. From the perspective of visual interpretation, a misclassification phenomenon obviously exists among the classification results of the experimental data; while after the process of removing the thin cloud-fog this paper proposes, the frustrating situation is improved to a great extent, which demonstrates that the proposed method not only improves the visual effects of the original imageries, but also raises the separability. As is shown in the original figure, thanks to the thin cloud-fog interference, the artificial buildings are enveloped in the plants. Though classification results of imageries of the other two methods witnesses a improvement, the effects are in significant when compared with the imageries in which the buildings are basically distinguished from plants, processed by the method this paper proposes.

\section{CONCLUSIONS}

The factors such as cloud-fog are contribute to the interpretation, analysis and utilization of optical remote sensing imageries. The current thin cloud-fog removal methods do an inferior job in eliminating the effects with the premise of retaining the spectral characteristics.

On the basis of previous research, this paper proposes the method based on stationary wavelet transformation and nonlinear gray-scale transformation. Stationary wavelet transformation has no needs to take a down-sampling, avoiding the errors of homomorphic filtering method caused by the frequency transformation and inverse transformation. Fully considering the difference of statistic characteristics of high resolution remote sensing imageries in different wavebrands areas or in non-cloud-fog-covered areas, ideal results are available because this method choose the corresponding filtering transformation or non-linear gray-scale transformation according to the real situation, which not only removes the noise from cloud-fog but also retains the light characteristics and space details of objects on the ground.

Addition, the classification results of the after-processed imageries also prove the fact that it is beneficial to the imageries classification and recognition with the thin cloudfog removal method this paper proposes.

\section{ACKNOWLEDGEMENT}

This research was funded by the civil Aerospace application project, a Remote Sensing Application Study Regarding the domestic satellites as primary data source', at the meantime, kindly assistance and support were also received from the Information Technology of Beijing Normal University in Zhuhai, Remote Sensing and Geographic Information System Society In Guangdong Province and student Zhang MengShi during the writing and experimenting process, highly appreciation for above all.

\section{REFERENCES}

[1] SONG Xiaoyu, LIU Liangyun, et al.Cloud removing based on single remote sensing image[J].Journal of OPTICAL TECHNIQUE,2006,32 (2:299-303(Ch).

[2] FENG Chun, MA Jianwen, DAI Qin, et al.AN IMPROVED METHOD FOR RAPID REMOVAL OF THIN CLOUD IN REMOTE SENSING IMAGES[J].Journal of REMOTE SENSING FOR LAND \& RESOURCES,2004,4(62):1-2(Ch).

[3] LI Yuechen, CHEN Jin, LIU Chunxia,et al.An effective approach to remove cloud2fog cover and enhance remote sensing imagery[J]. JOURNAL OF CHENGDU UNIVERSITY OF TECHNOLOGY (Science \& Technology Edition),2006,33(1):58-63(Ch).

[4] ZHAO Zhongming,ZHU Chongguang.Approach to Removing Cloud Cover from Satellite Imagery[J].Journal of REMOTE SENSING OF ENVIRONMENT CHINA,199611(3) ; 195-199(Ch).

[5] GUO Tongying, YOU Hongjian.Cloud Reduction of Multi2temporal Space2borne Remote Sensing Image Based on Wavelet Fusion[J].Journal of Survey and Mapping Notification,2007,3:40-42 (Ch).

[6] CAO Shuang.Research on the Cloud Removal Method of High Resolution Remote Sensing Images[D].Dissertation Submitted to Hohai University In fulfillment of the requirement For the degree of Master of Engineering,2006(Ch).

[7] CHEN Yao, WANG $\mathrm{J}$ in liang, LI Shihua.THEMETHODS FOR REMOVING THE EFFECTS OF CLOUD COVER IN REMOTE SENSING IMAGES[J].Journal of REMOTE SENSING FOR LAND \& RESOURCES,2006,1:61-65(Ch).

[8] Ying Zhang,Bert Guidon, and Josef Cihlar. An image transform to characterize and compensate for spatial variations in thin cloud contamination of Landsat images [J].Journal of Remote Sensing Environment, 2002, 82: 173-187.

[9] LI Cunjun1, LIU Liangyun, WAN GJihua,et al.Automatic detection and removal of thin haze based on own features of Landsat image[J].Journal of Zhejiang University ( Engineering Science),2006,40(1):0010-0013(Ch).

[10] LIU Yang,BAI Junwu. Research on the Cloud Removal Method of Remote Sensing Images[J].Journal of GEOMATICS \& SPATIAL INFORMATION TECHNOLOGY,2008,31(3):120-122(Ch)

[11] LU Li, WANG Yongtao, TIAN Jinwen1,et al.Cloud removal from satellite imagery based on SUSAN algorithm[J].Journal on Communications,2006,27(8):160-164(Ch).

[12] WEN Sha,YOU Zhisheng. A Performance Optimized Algorithm of Spatial Domain Homomorphic Filtering[J].Journal of APPLICATION RESEARCH OF COMPUTERS,2000,17(3):62-65(Ch).

[13] WU Chuanqing1, 2, WANG Qiao1, 3 , YANG Zhifeng.CloudMoving of Water RS Image Baed on $M$ ixed Pixel Model[J].JOURNAL OF REMOTE SENSING,2006,10(2) : 176-183 (Ch)

[14] HE Hui, PENG Wanglu, KUANG Jinyu.Thin Cloud Cover Removed from High-Resolution Remote Sensing Images Based on the Adaptive 
Filtering and Gray-scale Transformation[J].JOURNAL OF GEOINFORMATION SCIENCE,2009,11(3):305-311(Ch).

[15] SUN Yong-qiang, XU Wen-bo,SUN Jun. Research on image enhancement based on particle swarm optimization[J], Computer Engineering and applications, 2008 ,44(2):50-53.

[16] ZHOU Xiancheng, SHEN Quntai,WANG Junnian.Novel Image Contrast Transform Algorithm[J].SCIENCE TECHNOLOGY AND ENGINEERING,2007,7(21):5575-5579(Ch).

[17] FENG Chun.The Study of Atmospheric Correction Scheme Based on the Radiative Transfer Theory[D].Dissertation Submitted to China University of Geosciences(Beijing) for the degree of Doctor,2005

[18] Nason G P, Silverman B W. The stationary wavelet transform and some statistical applications[M]//Wavelets and statistics. Springer New York, 1995: 281-299.

[19] Sweldens W. The lifting scheme: A construction of second generation wavelets[J]. SIAM Journal on Mathematical Analysis, 1998, 29(2): $511-546$ 